Repository of the Max Delbrück Center for Molecular Medicine (MDC) Berlin (Germany)

http://edoc.molc-berlin.de/14510/

\title{
SILAC for biomarker discovery
}

Dittmar, G., Selbach, M.

This is the peer reviewed version of the following article: Dittmar, G., Selbach, M. SILAC for biomarker discovery. Proteomics Clinical Applications 9(3-4): 301-306, 2015.

which has been published in final form at http://dx.doi.org/10.1002/prca.201400112. This article may be used for non-commercial purposes in accordance with Wiley Terms and Conditions for Self-Archiving."

John Wiley \& Sons, Inc. 


\section{SILAC for biomarker discovery}

\section{Gunnar Dittmar and Matthias Selbach ${ }^{1}$}

Max Delbrück Center for Molecular Medicine, Robert-Rössle-Str. 10, 13092 Berlin, Germany

\section{Corresponding author:}

Gunnar Dittmar

Max Delbrück Centrum for Molecular Medicine

Robert-Rössle-Str. 10, D-13092 Berlin, Germany

Tel.: +493094062642

Fax.: +4930940649194

email: gdittmar@mdc-berlin.de

Matthias Selbach

Max Delbrück Centrum for Molecular Medicine

Robert-Rössle-Str. 10, D-13092 Berlin, Germany

Tel.: +493094063574

Fax.: +49309406 2394

email: matthias.selbach@mdc-berlin.de 


\author{
Abbreviations \\ CRP - C-reactive protein \\ HCV - hepatitis C virus \\ iTRAQ - Isobaric tags for relative and absolute quantitation \\ PrEST - Protein Epitope Signature Tags \\ PSA - prostate specific antigene \\ SIL - stable isotope labeling \\ SILAC - stable isotope labeling in cell culture \\ SRM - Selected reaction monitoring
}

\title{
Keywords
}

Quantification, mass spectrometry, quantitative proteomics, SILAC

Number or words: 3840

\section{Introduction}

"I often say that when you can measure what you are speaking about, and express it in numbers, you know something about it; but when you cannot measure it, when you cannot express it in numbers, your knowledge is of a meager and unsatisfactory kind [...]" (Lord Kelvin, Lecture on "Electrical Units of Measurement", 1883, published in Popular Lectures and Addresses, Vol. I, p. 73, Macmillan and Co, London/New York 1889).

Quantification is a particularly important aspect of biomarker discovery: Protein biomarkers such as prostate specific antigen (PSA) and C-reactive protein (CRP) are typically present in samples from both healthy and diseased donors[1]. Thus, biomarker discovery not only requires 
reliable protein identification but also quantification. The current trend of using collections or panels of several proteins as biomarkers further increases the need for accurate quantification.

Unfortunately, the intensity of a peak in a mass spectrum alone cannot be used to measure the absolute level of the corresponding peptide or protein. This is due to the fact that peak intensity depends on peptide-specific factors such as ionization efficiency. Thus, mass spectrometry-based proteomics is intrinsically not quantitative. However, over the last decade several strategies have been developed that enable quantification on a global proteomic scale[2-5]. The general principle of these strategies is that relative changes in the intensity of a peak rather than its absolute intensity are used for quantification. This can be done by comparing the intensities of the same peptide across different runs ("label free" quantification). Alternatively, peptides can be labeled with heavy stable isotopes ("stable isotope labeling" or SIL). Mass spectrometry can distinguish such heavy-labeled peptides from their normal (i.e. light) counterparts due to their mass difference, meaning that differentially-labeled samples can be mixed and analyzed together in a single run. This gives rise to pairs of physicochemically identical peptides of different isotope composition. The ratio of peak intensities of such peptide pairs accurately reflects relative changes in the abundance of the corresponding peptides. Due to the fact that samples are mixed before digestion, SIL-based approaches are more robust and generally considered more precise than label-free methods where samples are processed in parallel.

A particularly powerful approach for SIL-based quantification is stable isotope labeling with amino acids in cell culture (SILAC)[6]. Reviewing the huge field of SILAC-based proteomics is 
beyond the scope of this manuscript; we refer the interested reader to a number of excellent reviews[7-10]. Here, we will present selected examples on how SILAC-based strategies are currently used for biomarker discovery which we considered particularly informative. Since dozens of papers with this topic have been published our selection will inevitably be incomplete, and we apologize to the colleagues whose work could not be included. We will also discuss how SILAC can be used to obtain information about the absolute abundance of proteins - an aspect that is particularly relevant for mathematical modeling of diseases. Let us begin with a brief introduction to SILAC.

\section{A brief history of SILAC}

As a metabolic labeling technique, SILAC has its roots in the pioneering work of Rudolf Schoenheimer. He realized in 1935 that isotopes can be employed to label a physiological substance in such a way that the "the animal organism will not be able to differentiate between them" while the chemist is able to "distinguish and to estimate them in small quantities"[11] . Since then, growth media in which all atoms of a specific element are replaced by their heavy isotopes have been employed in many studies. For example, the Chait lab used ${ }^{15} \mathrm{~N}$ substituted medium to quantify phosphorylation in yeast [12] and the Heck lab established ${ }^{15} \mathrm{~N}$ labeling in two additional model organisms, C. elegans and D. melanogaster[13]. Although these labeling approaches allowed for global protein quantification in various model organisms, they also

have several disadvantages. First, ${ }^{15} \mathrm{~N}$-substituted media is difficult to make for mammalian cells that require more complex components. Second, since the mass shift introduced by SIL depends on the number of isotopically-altered elements in the analyte, it is typically different 
for different peptides, greatly complicating data analysis. Finally, due to the incorporation of multiple labels per analyte, even very highly enriched isotopes result in partial labeling. For example, consider a typical peptide with 20 nitrogen atoms: even $99 \%$ pure ${ }^{15} \mathrm{~N}$ will result in a labeling efficiency of only $82 \%\left(0.99^{20}\right)$, and this efficiency is further reduced for peptides with greater nitrogen content. These disadvantages pose a significant technical hindrance to biomarker discovery.

In 2002, Ong and colleagues developed SILAC as a simple and accurate approach to quantitative proteomics in cultured cells[6] which was later extended to complete organisms. In SILAC, tissue culture cells are labeled by cultivating them in growth medium containing heavy stableisotope encoded essential amino acids. Since cells lack the ability to synthesize these essential amino acids on their own, they are compelled to take them up from the growth medium and incorporate them into newly synthesized proteins. After about 8 cell divisions, virtually all cellular proteins are fully labeled. Differentially labeled samples are then combined, digested and analyzed by mass spectrometry. Lysine and arginine are the most popular amino acids for SILAC, since trypsin, the workhorse enzyme of proteomics, cleaves C-terminal of these residues, meaning most tryptic peptides will contain a single C-terminal label. The ratio of peak intensities between labeled peptide pairs forms the basis to accurately quantify relative changes in the abundance of the corresponding proteins (Figure $1 \mathrm{~A}$ ). Despite the enormous advantages of using the SILAC labeling technique, SILAC comes at a price. The use of two or three labels (light, medium, heavy) adds to the complexity of the sample; in the worst case triplicating the peaks detected from a proteome. This typically results in less identifications per 
run, although newest mass spectrometers are fast and sensitive enough to counteract this effect.

With more than 2,000 citations of the landmark SILAC paper, the method is one of the most popular approaches for expression proteomics, and has been employed to answer a wide range of questions in diverse areas of biomedical research[7]. The most significant advantage is that SILAC is more accurate than either label-free quantification or chemical labeling approaches[1416], due to the fact that differentially labeled samples can be combined very early in the workflow, minimizing errors introduced during sample handling. SILAC is particularly advantageous in situations where samples are extensively processed, such as fractionation and affinity-based enrichment of peptides with post-translational modifications. Furthermore, due to the high accuracy of SILAC, the method yields high quality data even for single peptides. This is particularly beneficial for analysis of posttranslational modifications which have to be quantified at the peptide level. For this reason, SILAC is particularly popular for phosphoproteomics[17-19]. Moreover, since SILAC is a metabolic labeling method, it can also be employed to quantify protein dynamics by measuring the rate of isotope incorporation. For example, dynamic SILAC can quantify protein turnover and pulsed SILAC can measure changes in protein synthesis on a proteome-wide scale[20-22].

\section{SILAC for patient-derived samples: the spike-in approach}

As the name indicates, SILAC was designed specifically to label cells in culture. However, metabolic labeling with amino acids can also be used to label entire organisms (for an overview see Kirchner and Selbach[23]). The approach has been used to create an entire SILAC zoo, ranging from lower eukaryotes 
like baker's yeast[24] via worms[25] and flies[14] up to mice [26]. Here, the naturally-occurring auxotrophies are used and the light lysine amino acid is replaced with its heavy counterpart. Long labeling times and special diet requirements preclude this technique from use on human beings, so alternate techniques must be used for the introduction of isotopic labels into human-derived samples. A number of post-lysis labeling methods have been well-established (e.g. dimethylation of lysines[27] or iTRAQ labeling of peptide amino groups[28]), although such approaches introduce significantly more variation into the system relative to metabolic labeling techniques, which allows mixing of the samples early in the biochemical preparation process. All variations in lysis efficiency, biochemical preparations etc. can thus be neglected[2,16].

Although SILAC cannot be used to directly label humans, the development of SILAC spike-in methods extend the use of the SILAC methods to non-cell culture samples of human origin, including clinical samples. For the spike-in approach, a human cell line is labeled with the SILAC amino acids in culture, then protein extracts of the labeled cells are subsequently spiked into the clinical human sample. The heavy labeled material is used as a reference standard (Figure 1B), whereby every identified protein is quantified in comparison to the standard, thus enabling relative quantification between the different samples. The idea of using cell culture-derived reference standards for quantification was first applied for quantitative proteomics in mouse brain[29] and has since become a popular experimental design for SILAC experiments.

The spike-in method, while allowing the quantification of patient samples, introduces more variability to the analysis in comparison to labeling in cell culture. Mixing the standard and the sample late in the preparation does not compensate for differences in lysis and extraction efficiency. In addition, the protein composition of the standard is itself problematic; in order to quantify the proteins present in the sample the protein has to be present in the labeled standard. If the protein is not detectable in the 
standard, SILAC-based quantification is impossible. Furthermore, SILAC is most accurate when the abundance of differentially labeled peptide pairs is similar i.e. same order of magnitude. The ideal reference standard, therefore, contains all proteins that are present in the clinical sample at an abundance similar to their physiological levels. These considerations led to the development of the Super-SILAC method[30]. Here the concept is that different cell lines are curated and combined in a way to best represent the sample of interest. In order to prepare the best possible reference standard several cell lines originating from different tissues are labeled and compared in an initial experiment with the complexity of the patient sample. The tailored combination of cell lines representing most of the proteins is used for the subsequent quantification steps. It is probably impossible or impractical to design a cell line reference standard that would be a perfect match to the tissue. However, the SuperSILAC approach has been shown to reduce the dynamic range of log2 fold changes between the reference standard and the tissue[30]. This increases the accuracy and precision of quantification, even though combining several cell lines increases the complexity of the heavy standard.

\section{SILAC and biomarkers: current applications}

Although the applications of SILAC-based proteomics for biomarker discovery are quite diverse, more than half of the studies focused on cancer. This is not surprising because early diagnosis is critical for the success of therapeutic intervention[31]. The human plasma proteome holds the promise of revolutionizing early diagnosis once the characteristic proteins indicative of a specific disease are known. However, protein concentrations in plasma span a dynamic range of more than ten orders of magnitude, posing a major challenge to proteomic discoveries[1]. This is probably reflected in the fact that no single biomarker identified and validated by proteomics has found its way into clinical practice[32]. A current trend is to focus on subproteomes such as secreted proteins (secretome), or on specific proteins of interest with targeted (as opposed to global) MS approaches. Rather than reviewing all of the different 
SILAC-based studies for biomarker discovery we will focus on the analysis of secretomes as well as targeted approaches.

Secreted proteins are particularly attractive for biomarker discovery. As their name indicates, these proteins are released from cells into the surrounding matrix and are thus detectable in biological fluids such as plasma. Additionally the secretion pattern is changed when a cell is transformed to a cancer cell[33]. So a number of studies used SILAC-based quantitative proteomics to identify proteins secreted from different kinds of cancer cells. For example, proteomic comparisons of neoplastic and nonneoplastic pancreatic cells led to the identification of more than 100 proteins that are preferentially secreted by neoplastic cells, with similar results obtained recently for gastric cancer[34,35]. SILAC-based proteomics was also used to characterize proteins secreted from metastatic and non-metastatic colon cancer cells[36]. In such studies, secreted proteins are identified from cell culture experiments and not directly in plasma. Instead, Yu and co-workers used secreted proteins derived from heavy-labeled pancreatic cancer cells to generate a reference standard[37]. Spiking this reference standard into sera from pancreatic cancer patients and into control sera allowed them to identify proteins upregulated in cancer. A study from the Mann lab combined the super-SILAC approach with biochemical enrichment of $\mathrm{N}$-glycosylated peptides from breast cancer cell lines[38]. Since the secretome profiles correctly clustered different cancer stages and corresponding glycopeptides were also detectable in human serum, this appears to be a promising strategy for finding the hallmarks of an underlying pathology. In a study using labeled cells as a spike-in standard, Montaldo et al. identified vimentin as a new biomarker for the diagnosis of liver fibrosis after HCV infection[39].

All techniques described so far are true shotgun proteomic techniques, capable of quantifying thousands of different proteins in one experiment. For many studies this is not really necessary, since the main targets are already well-characterized and the pathways involved have been identified. Here 
the combination of the SILAC labeling and immunoprecipitation techniques provides a unique approach to identify new biomarkers. Cell lines are labeled with the SILAC technique and the protein target of the analysis is precipitated using a specific antibody, with co-precipitated material then subjected to a shotgun mass spectrometric analysis. The aim is to identify the complete interactome of the target and the ratio of the heavy signal to the light one can be used to identify specific interactors vs. nonspecific binders. This allowed Paweletz and coworkers to identify new phosphorylations sites that are responsive to different PDK1 inhibitors[40]. In a related study, Andersen and colleagues identified 71 regulated Phospho-sites of the PI3K pathway using a similar approach[41].

Selected reaction monitoring (SRM) has been used as a method for absolute quantification of proteins[42], usually on a small scale due to the relative laborious setup of the technique in comparison to shot-gun based quantifications[43,44]. Here the known mass of a peptide is selected from the complex proteomic sample, fragmented followed by the selection of specific fragment ions. For the quantification of the target heavy labeled peptides are added to the proteomic preparation, serving as a reference of know concentration. A variation of this approach and an alternative to biochemical enrichment, SILAC-based quantification can also be used in SRM-type analysis, with a heavy SILAC spikein standard used as the reference in a classical SRM experiment. While the full potential of this approach remains to be explored, data from Liu and co-workers indicated that the method can be employed to reproducibly quantify 17 breast cancer-related focal adhesion proteins[15]. On the one hand, the advantage of this set-up is that SILAC can generate tens of thousands of isotopically labeled peptides at minimal costs. On the other, the method only allows for relative quantification, since the absolute amounts of the reference proteins spiked-in are unknown. A current development from the Uhlen and Mann lab uses a quantifiable library of tagged human protein fragments (termed Protein Epitope Signature Tags, PrEST) that were developed in the course of the Human Protein Atlas Project (Figure $1 \mathrm{C}$ ) $[45,46]$. The intelligent use of SILAC labeling and recombinant proteins, which are spiced into the 
proteomic mixture at known concentrations, allows the quantification of proteins in the proteomic sample. This enabled absolute quantification of 40 proteins in cancer cells. Applied to biomarker identification, this holds the promise of quantifying candidate proteins across a large number of samples with unprecedented accuracy. As Lord Kelvin suggested, expressing our knowledge of something in numbers is an overarching goal in our pursuit of understanding.

In conclusion, SILAC-based approaches have been successfully used in a number of biomarker studies. Compared to other methods, SILAC-based quantification has the unique advantage of low experimental variability. In the future, the widespread availability of high-resolution mass spectrometers and automated data processing pipelines will further advance biomarker discovery. Eventually, this may bring mass spectrometers to the doctor's office - the dream of early pioneers like John Fenn.

\section{Acknowledgement}

We thank Patrick Beaudette for reading the manuscript critically.

\section{Conflict of interest statement}

The authors have declared no conflict of interest.

\section{Literature}

[1] Anderson, N.L., The Human Plasma Proteome: History, Character, and Diagnostic Prospects. Molecular \& Cellular Proteomics 2002, 1, 845-867.

[2] Ong, S.-E., Mann, M., A practical recipe for stable isotope labeling by amino acids in cell culture (SILAC). Nat Protoc 2006, 1, 2650-2660.

[3] Gygi, S.P., Rist, B., Griffin, T.J., Eng, J., Aebersold, R., Proteome analysis of low-abundance proteins using multidimensional chromatography and isotope-coded affinity tags. J Proteome Res 2002, 1, 47-54.

[4] Gouw, J.W., Tops, B.B.J., Mortensen, P., Heck, A.J.R., Krijgsveld, J., Optimizing Identification and Quantitation of 15N-Labeled Proteins in Comparative Proteomics. Anal Chem 2008, 80, 7796-7803.

[5] Boersema, P.J., Aye, T.T., van Veen, T.A.B., Heck, A.J.R., Mohammed, S., Triplex protein quantification based on stable isotope labeling by peptide dimethylation applied to cell and tissue lysates. Proteomics $2008,8,4624-4632$. 
[6] Ong, S.-E., Blagoev, B., Kratchmarova, I., Kristensen, D.B., et al., Stable isotope labeling by amino acids in cell culture, SILAC, as a simple and accurate approach to expression proteomics. Mol Cell Proteomics 2002, 1, 376-386.

[7] Mann, M., Functional and quantitative proteomics using SILAC. Nat Rev Mol Cell Biol 2006, 7, 952-958.

[8] Ong, S.-E., Mann, M., Mass spectrometry-based proteomics turns quantitative. Nat Chem Bio/ 2005, 1, 252-262.

[9] Bantscheff, M., Lemeer, S., Savitski, M.M., Kuster, B., Quantitative mass spectrometry in proteomics: critical review update from 2007 to the present. Anal Bioanal Chem 2012, 404, 939-965.

[10] Ong, S.-E., The expanding field of SILAC. Anal Bioanal Chem 2012, 404, 967-976.

[11] Schoenheimer, R., Rittenberg, D., DEUTERIUM AS AN INDICATOR IN THE STUDY OF INTERMEDIARY METABOLISM. Science 1935, 82, 156-157.

[12] Oda, Y., Nagasu, T., Chait, B.T., Enrichment analysis of phosphorylated proteins as a tool for probing the phosphoproteome. Nat Biotechnol 2001, 19, 379-382.

[13] Krijgsveld, J., Ketting, R.F., Mahmoudi, T., Johansen, J., et al., Metabolic labeling of C. elegans and D. melanogaster for quantitative proteomics. Nat Biotechnol 2003, 21, 927-931.

[14] Sury, M.D., Chen, J.-X., Selbach, M., The SILAC fly allows for accurate protein quantification in vivo. Mol Cell Proteomics 2010, 9, 2173-2183.

[15] Liu, N.Q., Dekker, L.J.M., Stingl, C., Güzel, C., et al., Quantitative proteomic analysis of microdissected breast cancer tissues: comparison of label-free and SILAC-based quantification with shotgun, directed, and targeted MS approaches. J Proteome Res 2013, 12, 4627-4641.

[16] Lau, H.-T., Suh, H.W., Golkowski, M.G., Ong, S.-E., Comparing SILAC and dimethyl labeling approaches for quantitative proteomics. J Proteome Res 2014.

[17] Gruhler, A., Olsen, J.V., Mohammed, S., Mortensen, P., et al., Quantitative phosphoproteomics applied to the yeast pheromone signaling pathway. Mol Cell Proteomics 2005, 4, 310-327.

[18] Olsen, J.V., Blagoev, B., Gnad, F., Macek, B., et al., Global, in vivo, and site-specific phosphorylation dynamics in signaling networks. Cell 2006, 127, 635-648.

[19] Dephoure, N., Zhou, C., Villén, J., Beausoleil, S.A., et al., A quantitative atlas of mitotic phosphorylation. Proc Natl Acad Sci USA 2008, 105, 10762-10767.

[20] Schwanhäusser, B., Gossen, M., Dittmar, G., Selbach, M., Global analysis of cellular protein translation by pulsed SILAC. Proteomics 2009, 9, 205-209.

[21] Schwanhäusser, B., Busse, D., Li, N., Dittmar, G., et al., Global quantification of mammalian gene expression control. Nature 2011, 473, 337-342.

[22] Doherty, M.K., Hammond, D.E., Clague, M.J., Gaskell, S.J., Beynon, R.J., Turnover of the human proteome: determination of protein intracellular stability by dynamic SILAC. J Proteome Res 2009, 8 , 104-112.

[23] Kirchner, M., Selbach, M., In vivo quantitative proteome profiling: planning and evaluation of SILAC experiments. Methods Mol Biol 2012, 893, 175-199.

[24] de Godoy, L.M.F., Olsen, J.V., Cox, J., Nielsen, M.L., et al., Comprehensive mass-spectrometry-based proteome quantification of haploid versus diploid yeast. Nature 2008, 455, 1251-1254.

[25] Larance, M., Bailly, A.P., Pourkarimi, E., Hay, R.T., et al., Stable-isotope labeling with amino acids in nematodes. Nat Methods 2011.

[26] Krüger, M., Moser, M., Ussar, S., Thievessen, I., et al., SILAC mouse for quantitative proteomics uncovers kindlin-3 as an essential factor for red blood cell function. Cell 2008, 134, 353-364.

[27] Boersema, P.J., Raijmakers, R., Lemeer, S., Mohammed, S., Heck, A.J.R., Multiplex peptide stable isotope dimethyl labeling for quantitative proteomics. Nat Protoc 2009, 4, 484-494.

[28] Ross, P.L., Huang, Y.N., Marchese, J.N., Williamson, B., et al., Multiplexed protein quantitation in Saccharomyces cerevisiae using amine-reactive isobaric tagging reagents. Mol Cell Proteomics 2004, 3, 1154-1169.

[29] Ishihama, Y., Oda, Y., Tabata, T., Sato, T., et al., Exponentially modified protein abundance index (emPAl) for estimation of absolute protein amount in proteomics by the number of sequenced peptides per protein. Mol Cell Proteomics 2005, 4, 1265-1272.

[30] Geiger, T., Cox, J., Ostasiewicz, P., Wiśniewski, J.R., Mann, M., Super-SILAC mix for quantitative proteomics of human tumor tissue. Nat Methods 2010, 7, 383-385. 
[31] Richards, M.A., The size of the prize for earlier diagnosis of cancer in England. Br. J. Cancer 2009,101 Suppl 2, S125-9.

[32] Anderson, N.L., The clinical plasma proteome: a survey of clinical assays for proteins in plasma and serum. Clin. Chem. 2010, 56, 177-185.

[33] Villarreal, L., Méndez, O., Salvans, C., Gregori, J., et al., Unconventional secretion is a major contributor of cancer cell line secretomes. Mol Cell Proteomics 2013, 12, 1046-1060.

[34] Grønborg, M., Kristiansen, T.Z., Iwahori, A., Chang, R., et al., Biomarker discovery from pancreatic cancer secretome using a differential proteomic approach. Mol Cell Proteomics 2006, 5, 157-171.

[35] Marimuthu, A., Subbannayya, Y., Sahasrabuddhe, N.A., Balakrishnan, L., et al., SILAC-based quantitative proteomic analysis of gastric cancer secretome. Proteomics Clin Appl 2013, 7, 355-366.

[36] Barderas, R., Mendes, M., Torres, S., Bartolomé, R.A., et al., In-depth characterization of the secretome of colorectal cancer metastatic cells identifies key proteins in cell adhesion, migration, and invasion. Mol Cell Proteomics 2013, 12, 1602-1620.

[37] Yu, K.H., Barry, C.G., Austin, D., Busch, C.M., et al., Stable isotope dilution multidimensional liquid chromatography-tandem mass spectrometry for pancreatic cancer serum biomarker discovery. J Proteome Res 2009, 8, 1565-1576.

[38] Boersema, P.J., Geiger, T., Wiśniewski, J.R., Mann, M., Quantification of the N-glycosylated secretome by super-SILAC during breast cancer progression and in human blood samples. Mol Cell Proteomics 2013, $12,158-171$.

[39] Montaldo, C., Mattei, S., Baiocchini, A., Rotiroti, N., et al., Spike-in SILAC proteomic approach reveals the vitronectin as an early molecular signature of liver fibrosis in hepatitis $\mathrm{C}$ infections with hepatic iron overload. Proteomics 2014, 14, 1107-1115.

[40] Paweletz, C.P., Andersen, J.N., Pollock, R., Nagashima, K., et al., Identification of Direct Target Engagement Biomarkers for Kinase-Targeted Therapeutics. PLoS ONE 2011, 6, e26459.

[41] Andersen, J.N., Sathyanarayanan, S., Di Bacco, A., Chi, A., et al., Pathway-based identification of biomarkers for targeted therapeutics: personalized oncology with PI3K pathway inhibitors. Sci Transl Med 2010, 2, 43ra55.

[42] Picotti, P., Bodenmiller, B., Mueller, L.N., Domon, B., Aebersold, R., Full Dynamic Range Proteome Analysis of S. cerevisiae by Targeted Proteomics. Cell 2009, 138, 795-806.

[43] Bagola, K., Delbrück, von, M., Dittmar, G., Scheffner, M., et al., Ubiquitin binding by a CUE domain regulates ubiquitin chain formation by ERAD E3 ligases. Molecular Cell 2013, 50, 528-539.

[44] Hinz, M., Stilmann, M., Arslan, S.C., Khanna, K.K., et al., A cytoplasmic ATM-TRAF6-clAP1 module links nuclear DNA damage signaling to ubiquitin-mediated NF-KB activation. Molecular Cell 2010, 40, 63-74.

[45] Persson, A., Hober, S., Uhlen, M., A human protein atlas based on antibody proteomics. Curr. Opin. Mol. Ther. 2006, 8, 185-190.

[46] Zeiler, M., Straube, W.L., Lundberg, E., Uhlen, M., Mann, M., A Protein Epitope Signature Tag (PrEST) library allows SILAC-based absolute quantification and multiplexed determination of protein copy numbers in cell lines. Mol Cell Proteomics 2012, 11, 0111.009613.

Figure 1 legend

A. SILAC labeling and analysis of cells. Starting from an unlabeled culture, cells are labeled by cultivating them in media containing SILAC amino acids. The cells are harvested and combined prior to lysis and protein extraction. After digesting the proteins with a suitable protease, the resulting peptides are subjected to chromatographic separation and analyzed on a high-resolution mass 
spectrometry. The signal in the MS spectra is then used to quantify the amount of peptides present in the sample. B. Spike-in SILAC analysis. In a separate preparation a cell line is labeled with heavy amino acids, and the extracted proteins destined for use as a reference standard. The same amount of the heavy protein standard is spiked into protein extracts prepared from the sample of interest. After digestion with a suitable protease the peptides are purified and analyzed on a high-resolution mass spectrometer. The isotopically-heavier peak of the spiked-in material (red) is used as an internal standard, and the corresponding isotopically-light peptide is quantified in relation to this standard, allowing the comparison between the different samples. C. Absolute quantification using PrEST standard proteins has several steps. The first is the recombinant expression and quantification of the PrEST standard protein in E.coli. The PrEST epitope tag contains several domains, among them an albumin binding domain (ABP or ProA). A heavy-labeled recombinant version of the ABP proteins is then used for the quantification of the PrEST-tagged recombinant protein. The absolute amount of the $A B P$ is determined by amino acid analysis. By determining the relative ratio of the PrEST tag in relation to the $A B P$, the absolute amount of the PrEST-tagged protein is determined. In a second step a known amount of the PrEST-tagged protein is spiked into the sample of interest. By measuring the relative ratio of the peptides derived from the PrEST protein to the cellular protein, the absolute abundance of the proteins can be calculated. 
A

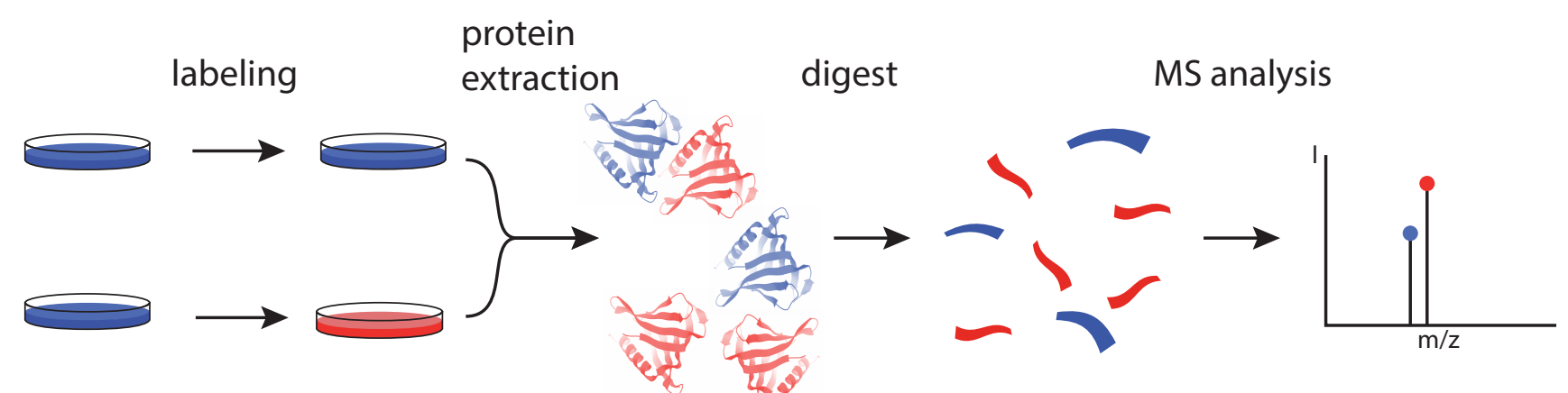

B

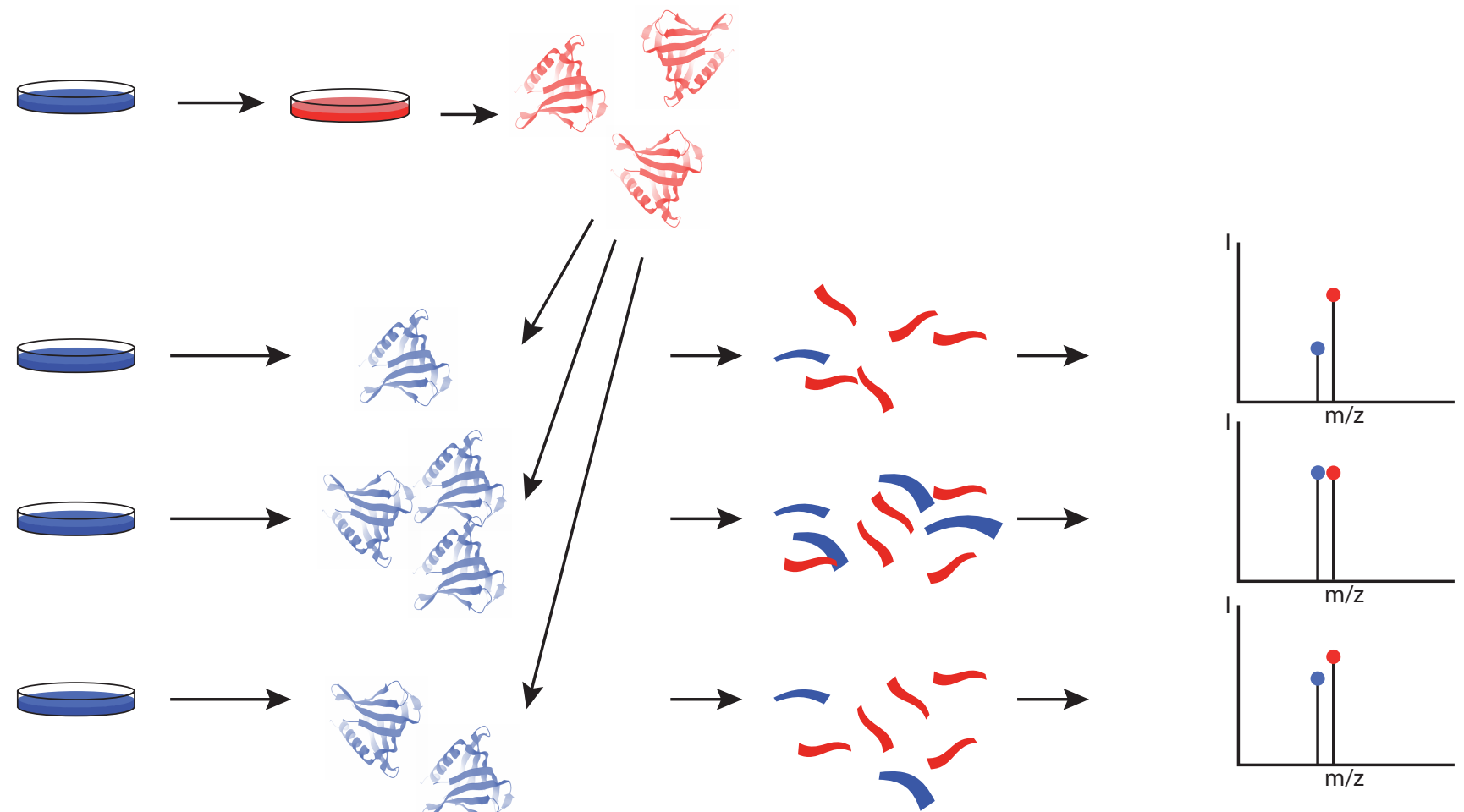

C
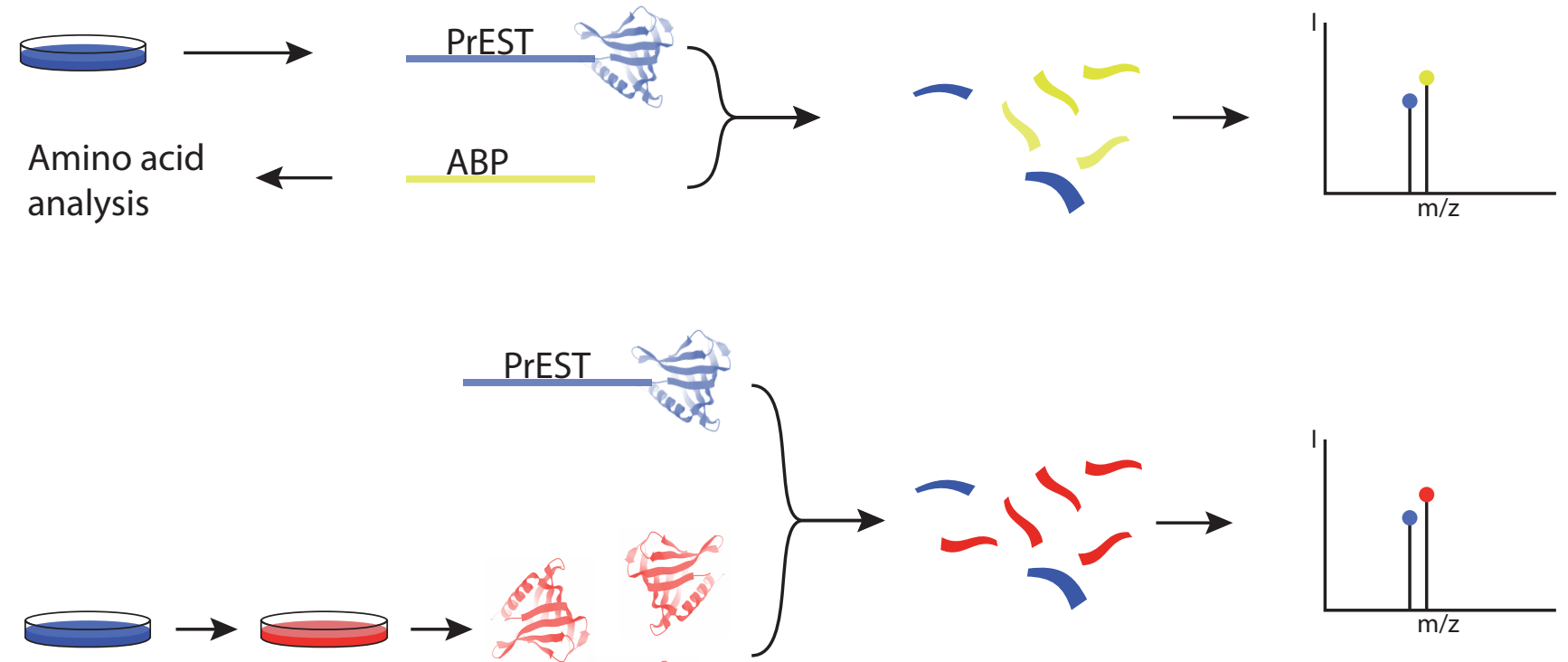